\title{
Programação da Produção e Meta- Heurísticas: Uma Revisão de Mapeamento Sistemático
}

\section{Ailton Silva Ferreira}

ailtonsilvaferreira@yahoo.com.br
Universidade Estadual do Norte Universidade Estadual do Norte
Fluminense (UENF), Macaé, Rio de Fluminense

Cristiano Manhães de Oliveira cristianomo@pg.uenf.br

Universidade Estadual do Norte Fluminense (UENF), Campos dos Goytacazes, Rio de Janeiro, Brasil

Hussein Adnen Mustafa Hussein.16@hotmail.com Universidade Estadual do Norte Fluminense (UENF), Campos dos Goytacazes, Rio de Janeiro, Brasil

Thiago Dan Said thiagosaid@pq.uenf.br Universidade Estadual do Norte Fluminense (UENF) Goytacazes, Rio de Janeiro, Brasil

\section{Tiago Andrade Muniz Terra} tiagoterra@pq.uenf.br

Universidade Estadual do Norte Fluminense (UENF), Campos dos Goytacazes, Rio de Janeiro, Brasil
A Programação da Produção tem recebido atenção de pesquisadores da área de Pesquisa Operacional desde a década de 50 e um grande número de trabalhos foi desenvolvido desde então, da mesma forma, devido à Internet, o rastreamento bibliográfico de um campo de pesquisa está vez mais demorado, como também encontrar relações entre autores e publicações relevantes, está ficando cada vez mais difícil, devido ao crescente volume de informações. Dessa forma, este trabalho busca realizar uma revisão de mapeamento sistemático, com relação à programação da produção e algumas das principais meta-heurísticas (Hill Climbing, Simulated Annealing,Tabu Search,Harmony Search, GRASP, Evolutionary Algorithms, Genetic Algorithms, Evolution Strategies, Genetic Programming, Differencial Evolution, Evolutionary Programming, Particle Swarm Optimization, Memetic Algorithms e Ant Colony), através de indicadores bibliométricos e análise de redes (Page Rank), busca-se demonstrar a relação de artigos, autores e publicações mais importantes e de maior prestígio de dados originados da base Web of Science com auxílio das ferramentas NAILS e GEPHI. Finalmente, é demonstrado que a utilização de meta-heurísticas na Programação da Produção, apesar de ser uma linha de pesquisa antiga, ainda se mantém com grande interesse e em constante desenvolvimento.

PALAVRAS-CHAVE: Programação da Produção. Meta-Heurísticas. Análise de Redes. 


\section{INTRODUÇÃO}

Revisões de Mapeamento Sistemático (EMS) são usadas para estruturar uma área de pesquisa, enquanto que as revisões sistemáticas focam em reunir e sintetizar (PETERSEN; VAKKALANKA; KUZNIARZ, 2015). Existe um número cada vez maior de artigos sendo publicado, o que torna o rastreamento bibliográfico cada vez mais demorado, por outro lado, os EMS estão se tornando um método cada vez mais importante de obter o estado de um campo de pesquisa (KNUTAS et al., 2015).

A extração de dados de produção acadêmica, identificação de padrões bibliométricos, modelagem e visualização das redes de interação entre coautores são tópicos relevantes na área de Bibliometria e Cientometria (MENA-CHALCO; JUNIOR, 2009). A Análise de Redes Sociais (ARS), não é uma teoria formal em sociologia, mas uma estratégia para investigar as estruturas sociais, onde são estudadas as redes de colaboração, além de outras formas de iteração das redes sociais, além disso, a Internet representa uma rede social de uma escala sem precedentes (OTTE; ROUSSEAU, 2002).

Entretanto, hoje em dia, com advento da Internet, encontrar relações entre autores e publicações relevantes, está ficando cada vez mais difícil, devido ao crescente volume de informações (KLINK et al., 2006). As redes de material acadêmico têm grande quantidade de informações. Aproximadamente 114 milhões de documentos científicos (tais como livros, artigos, teses, dissertações e documentos técnicos), somente em inglês, são hoje acessíveis pela web (KHABSA; GILES, 2014).

A utilização da teoria dos grafos para a representação das redes sociais possui algumas vantagens, tais como: representar uma rede social, mensurar precisamente importantes propriedades estruturais, além disso, a representação visual de um grafo permite aos pesquisadores analisarem as interações sociais, descobrir padrões que não foram detectados anteriormente (WASSERMAN; FAUST, 1994). Alguns aplicativos e bibliotecas possuem a capacidade de fornecer uma análise de redes sociais, tais como o Gephi (FAHIMNIA; SARKIS; DAVARZANI, 2015).

Indicadores bibliométricos de ligação e a ARS vêm se consolidando como ferramentas de estudo e de amplo entendimento de fenômenos sociais, como redes de co-citações, estruturas de colaboração científica, redes temáticas de produção científica e outras formas de redes de interação social (OTTE; ROUSSEAU, 2002).

O rankeamento de autores é de vital importância para identificar o impacto de um pesquisador num determinado campo científico, para isso existem vários métodos tais como número de citações, $h$-index PageRank e weighted PageRank (DING, 2011a). Dentre esses métodos, o número de citações proposto por Garfield (1955) é o mais comum para mensurar a importância de publicações científicas (MASLOV; REDNER, 2008) e juntamente com journal impact factor, tornou-se um indicador estabelecido para avaliar o impacto de artigos publicados em um periódico (YAN; DING, 2010). Entretanto, o impactfactor, e $h$-index usam contagens de citações e não distinguem citações provenientes de diferentes autores, nem as características das redes de citação (DING, 2011b). 
Bibliometricamente a popularidade, pode ser mensurada como o número de vezes que um autor é citado e prestígio como o número de vezes que um autor é citado em trabalhos altamente citados (DING; CRONIN, 2011), como demonstrado na Figura 1.

Figura 1: Prestígio (citados por artigos altamente citados) e popularidade (citados por artigos normais)

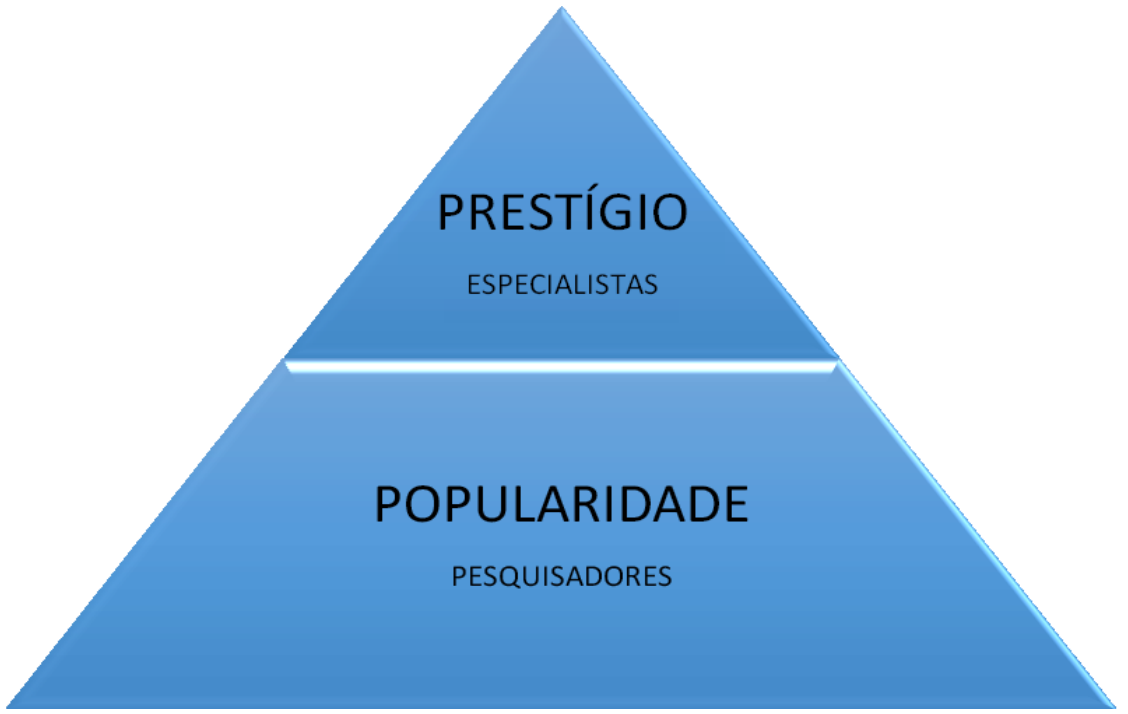

Fonte: Adaptado de (DING, 2011b).

Um artigo altamente citado pode não ser necessariamente um trabalho de prestígio embora em alguns casos possa haver uma forte correlação positiva entre as duas medidas (FAHIMNIA; SARKIS; DAVARZANI, 2015). O uso de citações como métrica de importância possui algumas deficiências como, por exemplo, a que artigos antigos, porém inovadores, são pouco citados devido à uma comunidade científica menor quando foram publicados, além do fato que publicações sobre descobertas científicas significativas deixam de ser citadas quando seus resultados são incorporados a livros (MASLOV; REDNER, 2008).

O PageRank calcula a importância de uma página web através da suas conexões com outras páginas (BRIN; PAGE, 1998), evita parcialmente tais deficiências, dando maior peso às publicações que são citadas por trabalhos importantes e também identificando um grande número de artigos que contêm resultados inovadores (MASLOV; REDNER, 2008).

Durante pelo menos cinquenta e cinco anos, a pesquisa em ciência da computação levou ao desenvolvimento de muitos Algoritmos que resolvem determinadas classes de problemas para a otimização (WEISE, 2011). Devido ao grande número de trabalhos desenvolvidos essa pesquisa busca realizar um Estudo de Mapeamento Sistemático, com auxílio da ferramenta NAILS (KNUTAS et al., 2015)para compilação dos dados originados na base Web of Science e geração de relatórios estatísticos e posterior ARS usando o GEPHI, na análise de rede é utilizado o PageRank.

O objetivo desse artigo busca realizar uma revisão de mapeamento sistemático, com relação à programação da produção- PP e algumas das principais meta-heurísticas, através da realização de uma bibliometria. 
Este trabalho está desenvolvido como segue. Primeiramente, na Seção 2, apresentam-se alguns conceitos sobre meta-heurísticas e suas aplicações com relação à PP. Na Seção 3, apresenta-se a metodologia. Já na Seção 4, apresentamse os principais resultados. Por fim, apresentam-se as conclusões na Seção 5.

\section{PP E META-HEURÍSTICAS}

A Programação da Produção (Scheduling ou PP) pode ser definida como a alocação de recursos ao longo do tempo para executar tarefas (MACCARTHY; LIU, 1993),trata-se de uma área da Pesquisa Operacional (PO), que consiste da organização de tarefas a serem processadas em uma ou várias máquinas que se baseia na otimização combinatória, para resolver uma série de problemas (YALAOUI et al., 2010).

PP tem recebido atenção de pesquisadores da área de PO desde a década de 50 e um grande número de trabalhos foram desenvolvido desde então (MACCARTHY; LIU, 1993). Antes da explanação de algumas das técnicas que foram desenvolvidas no decorrer desses anos, alguns conceitos devem ser esclarecidos (YALAOUI et al., 2010):

- Job: é uma tarefa, que possui um conjunto de informações (tempos de processamento, de conclusão) e geralmente é composta de várias operações sucessivas;

- Recurso: são os meios de produção (máquinas);

- Restrição: uma condição necessária para a realização dos jobs;

- Critério: é o objetivo a ser otimizado, como por exemplo, minimização do makespan;

Os problemas de scheduling podem ser classificados como (MACCARTHY; LIU, 1993):

1. Job shop: Cada job tem seu de fluxo ou rota específica através das máquinas;

2. Flow shop: Cada job tem um padrão de fluxo idêntico;

3. Open shop: não existe um padrão de fluxo específico para os jobs;

4. Permutationflow shop: possui um fluxo no qual a ordem de processamento de trabalhos em todas as máquinas é a mesma;

5. Single machine shop: Apenas uma máquina é disponível;

6. Máquinas paralelas: $K$ máquinas idênticas em uma única fase de processamento. Cada job precisa de uma dessas máquinas;

7. Job shop com máquinas paralelas: Um job shop em que existem $\mathrm{k}$ máquinas idênticas em cada estágio $(i=1, \ldots, m)$ e qualquer job que exija essa etapa precisa ser processado em uma dessas máquinas.

Para a obtenção de soluções para tais problemas a PO, aplica dois princípios: métodos exatos que fornecem a melhor solução, mas resolve apenas problemas pequenos e métodos aproximados que fornecem boas soluções. Essa 
segunda categoria existe vários métodos que podem ser divididos em heurísticas e meta-heurísticas (YALAOUI et al., 2010). Heurísticas e meta-heurísticas.

O desenvolvimento e sucesso de heurísticas, especialmente as meta heurísticas, foi de grande interesse dos pesquisadores na década de 90 na aplicação desses métodos em problemas de otimização combinatória multiobjectivo considerados difíceis computacionalmente (EHRGOTT; GANDIBLEUX, 2000), as heurísticas podem ser classificadas em (ARROYO, 2002):

- Heurísticas construtivas: são especializadas para um dado problema e constroem uma solução pela adição de componentes da mesma através de regras específicas associadas com a estrutura do problema;

- Heurísticas de busca local ou em vizinhança: iniciam com uma solução completa do problema, e constroem uma vizinhança desta solução, em que contém todas as soluções alcançáveis. Dessa vizinhança, escolhe-se uma solução que seja melhor que a solução inicial. A solução escolhida torna-se a nova solução inicial e o processo continua até encontrar um ótimo local;

- Meta heurísticas: são métodos inteligentes flexíveis, pois possuem uma estrutura com componentes genéricos que são adaptados ao problema que se quer resolver, na Error! Reference source not found. são exemplificadas algumas das principais meta heurísticas.

Figura 2 - Exemplo de figura

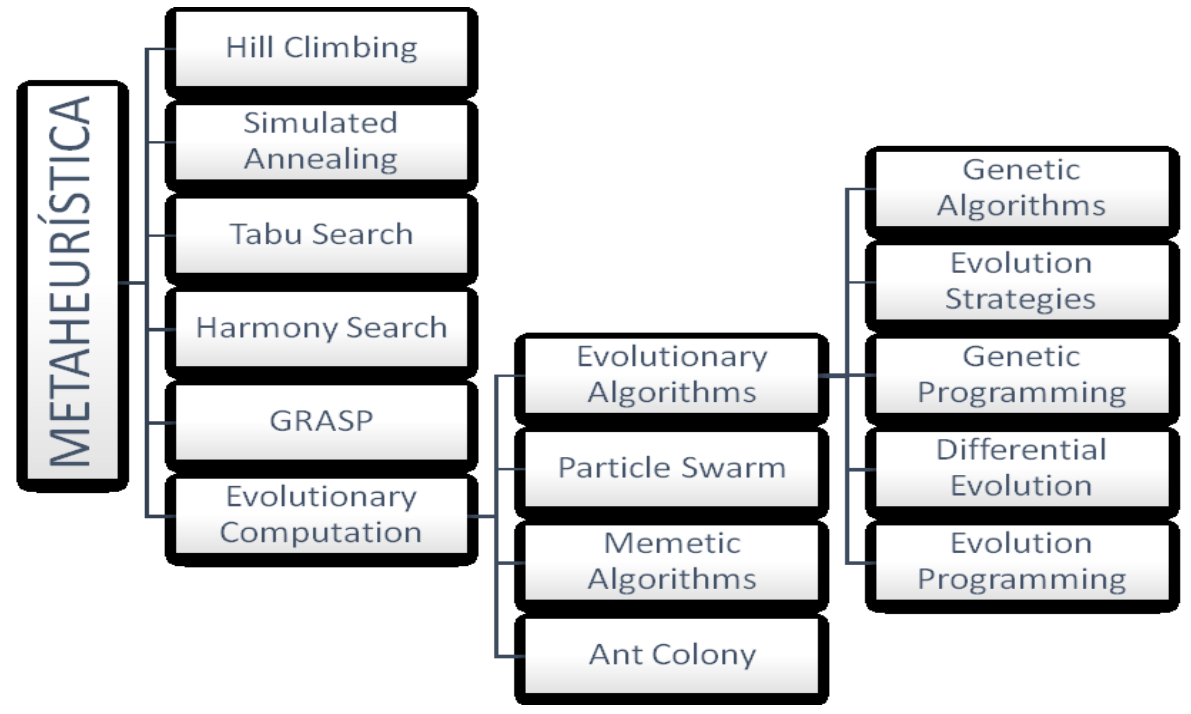

Fonte: UTFPR (2017) 


\section{HILL CLIMBING}

É um método de busca local que utiliza um procedimento de melhora iterativa (iterative improvement), ou seja, inicia com uma solução arbitrária do problema e segue iterativamente tentando encontrar um máximo ou mínimo (ótimo local) de uma função objetivo (RUSSELL; NORVIG, 1995).

Em Miller et al.(1999) um Algoritmo Genético híbrido é proposto para o problema de programação de uma máquina, onde para melhorar sua eficiência e desempenho, o Hill Climbing é unido ao Algoritmo Wagner-Whitin (WAGNER; WHITIN, 1958).

\section{SIMULATED ANNEALING}

Para Raaymakers e Hoogeveen (2000), Simulated Annealing é um algoritmo de pesquisa de aleatória de vizinhança que aceita soluções com um custo mais alto mas com certa probabilidade. Os autores enfatizam que escolheram esse método em seu trabalho pois provou ser um procedimento de pesquisa local eficaz podendo ser facilmente aplicado a diferentes tipos de problemas.

\section{TABUSEARCH}

O método Tabu Search- TS foi proposto por (GLOVER, 1986) e, segundo o autor, pode ser visto como uma "meta-heurística" sobreposta em outra heurística, ou seja, uma meta-heurística que orienta um procedimento de pesquisa heurística local para explorar o espaço da solução além da otimização local (GLOVER; LAGUNA; MARTI, 2007). O método tem como base a premissa de que a resolução de problemas, para se qualificar como inteligente, deve incorporar memória adaptativa e exploração responsiva (GLOVER; MARTí, 1986).

Tang, Wang e Liu (2008) aplicaram o método Tabu Search em uma indústria siderúrgica com o objetivo de resolver um problema de programação em larga escala denominado color-coating production scheduling for coils in inventory (CCPSCI), os autores optaram pelo TS devido ao método ter sido aplicado com sucesso para resolver problemas de otimização combinatória na indústria siderúrgica em outros casos.

\section{HARMONYSEARCH}

O algoritmo HarmonySearch (HS), foi inspirado no processo de desempenho musical natural quando um músico procura um melhor estado de harmonia (ZONG WOO GEEM; JOONG HOON KIM; LOGANATHAN, 2001). É uma das mais recentes heurísticas evolutivas baseadas em população, semelhante ao AG, o HS gera uma nova solução candidata, de todas as soluções existentes e comparações numéricas (WANG; PAN; TASGETIREN, 2010).

Em Wang, Pan e Tasgetiren (2010), três algoritmos HS híbridos são desenvolvidos para resolver o problema de Flow Shop Scheduling with blocking para minimizar o tempo de fluxo total. 
GRASP ou Greedy Randomized Adaptive Search Procedures, conforme aponta (MONKMAN; MORRICE; BARD, 2008), foi desenvolvida por Feo e Bard (1989) sendo detalhada em (FEO; VENKATRAMAN; BARD, 1991)(FEO; RESENDE, 1995).

O método combina o poder de heurísticas ganaciosas, aleatorização e técnicas convencionais de busca local, como procedimento de troca. O método é repetitivo e cada repetição é composta por 2 fases, uma de construção e uma de busca local, sendo que a primeira constrói inteligentemente uma solução para o problema e o segundo aplica uma técnica de busca à solução construída para encontrar um ótimo local. (FEO; RESENDE, 1995; FEO; VENKATRAMAN; BARD, 1991)

Heath, Bard e Morrice (2012) aplicam o método GRASP na indústria eletrônica e destacam que nos últimos 20 anos, o GRASP provou ser útil em uma ampla variedade de aplicações, incluindo roteamento, sequenciação e agendamento de veículos, telecomunicações e mapeamento genético.

\section{ALGORITMOS EVOLUTIVOS (EVOLUTIONARY ALGORITHMS)}

Basicamente, Vidal et al. (2011) destaca que os Algoritmos Evolutivos (AE) são utilizados com freqüência para resolver problemas programação da produção do tipo job-shop (Job-Shop SchedulingProblems - JSSP). Tal afirmação se dá porque este método, geralmente, obtém resultados melhores que abordagens tradicionais. O termo "evolutivos" envolve alguns métodos de pesquisa que tem como base os processos de evolução da natureza (VIDAL et al., 2011).

\section{ALGORITMOS GENÉRICOS (GENETIC ALGORITHMS)}

Os Algoritmos Genéticos (AG), são classificados como um dos AE mais eficientes na utilização da abordagem elitista (VIDAL et al., 2011). Os primeiros trabalhos relacionados a AG surgiram por volta de meados da década de 1950, com aplicações de simulações auxiliadas por computadores para obter maior proximidade com processos genéticos (BARRICELLI, 1962; FRASER, 1957). O termo Algoritmo Genético foi introduzido por Bagley (1967).

Em Vidal et al. (2011) os Algoritmos Genéticos são combinados com Redes Neurais, a fim de tratar um problema JSSP em máquinas, sendo realizado em uma indústria de móveis personalizados.

\section{ESTRATÉGIAS EVOLUTIVAS (EVOLUTION STRATEGIES)}

Hicks, Song e Earl (2007) utilizam as Estratégias Evolutivas (EE) para localizar uma solução global ou quase ótima, dessa forma, o método busca otimizar um determinado objeto de estudo de pesquisa estocástica com base nos princípios da evolução biológica. Tal método é parecido com os Algoritmos Genéticos por conter operadores de seleção, recombinação / crossover e de mutação. 
Schwefel (1995) e Schwefel\& Back (1998) destacam que as EE são mais adequadas para serem usadas em problemas de otimização numérica, por usar variáveis continuas na representação de uma solução. Em seu trabalho, Schwefel\& Back (1998) utilizam métodos heurísticos e de Estratégias Evolutivas para tratar problemas de programação dinâmica de produtos complexos que são processados em diversos níveis de produção e montagem com capacidade finita.

\section{PROGRAMAÇÃO GENÉTICA (GENETIC PROGRAMMING)}

Dentro dos algoritmos evolutivos, a Programação Genética (PG) é tratada como especial, nela os candidatos a solução são representados por estruturas de árvores com tamanhos variados. Pode-se destacar a PG como um método muito promissor se utilizado como hiper-heurístico gerando regras de despacho (PICKARDT et al., 2010).

Em seu artigo, Pickardtet al. (2010), procura gerar regras de despacho para uma fábrica de semicondutores com a combinação de simulação a eventos discretos e Programação Genética. Os autores destacam que os resultados são impressionantes, podendo reduzir o atraso médio ponderado em até $70 \%$.

\section{PROGRAMAÇÃO DIFERENCIAL (DIFFERENCIAL EVOLUTION)}

Trata-se de um método estocástico que se baseia em uma população e um otimizador global efetivo em um domínio contínuo (STORN; PRICE, 1997). Os algoritmos Evolução Diferencial (ED), são constantemente utilizados para resolução de problemas contínuos, porém não pode ser aplicado diretamente para problemas de flow shop em domínios discretos (SHAO; PI, 2016).

\section{PROGRAMAÇÃO EVOLUTIVA (EVOLUTION PROGRAMMING)}

O método da Programação Evolutiva está, geralmente, relacionado com as teorias da Estratégia da Evolução, otimizando vetores e números reais. Ao longo do tempo, o método foi mais utilizado na derivação de estruturas parecidas com algoritmos, podendo citar as máquinas de estados finitos (WEISE, 2011).

\section{PARTICLE SWARM OPTIMIZATION}

Proposto pela primeira vez por (EBERHART; KENNEDY, 1995; KENNEDY; EBERHART, 1995), Particle Swarm Optimization é uma técnica de computação evolutiva através de melhorias individuais, mais a cooperação e competição da população (LIU; WANG; JIN, 2007).

Conforme apontam Khan e Niemann-Delius (2014), o PSO é inspirado na naturaza, se baseando na interação social de indivíduos que vivem juntos em grupos, por exemplo, bandos de pássaros, cardumes de peixes, rebanhos de animais, entre outros. Os autores complementam que o algorítmo executa o processo de busca usando uma população (enxame) de indivíduos (partículas), sendo que cada indivíduo é uma solução potencial para o problema de otimização. 
Com objetivo de resolver problema de no-waitflow shop scheduling Liu, Wang e Jin (2007) utilizam um algoritmo híbrido baseado no PSO. Já Khan e Niemann-Delius (2014) utilizaram o algoritmo para o sequenciamento de extração de minas.

\section{MEMETIC ALGORITHMS}

De acordo com Moscato e Cotta (2003) os algoritmos meméticos tiveram origem no final dos anos 80 , no entanto, outros trabalhos em anos anteriores já apresentavam características semelhantes, sendo classificados inicialmente como algoritmos genéticos híbridos.

Para Liu et al. (2013) os algoritmos meméticos podem ser considerados como a união de uma busca global baseada em população e melhorias locais resultando em ferramentas de otimização mais robustas e efetivas, segundo o autor, esse algoritmo também é chamado de algoritmo heurístico hibrido.

Geismar et al. (2008) apontam que, em geral, a ordem de processamento do algoritmo memético é a mesma que a do algoritmo genético, destacando que a principal diferença em suas implementações é que o passo de mutação aleatória é substituído por uma pesquisa local que tenta muitas trocas de elementos diferentes dentro do indivíduo na tentativa de melhorar sua permutação.

\section{ANT COLONY}

O algorítmo Ant Colony foi desenvolvida por Dorigo (1992) inspirado pela observação de colônias de formigas reais, observando como as formigas podem encontrar o caminho de rota mais curto de sua colônia para uma fonte de alimento e vice-versa.

Atighehchian, Bijari e Tarkesh (2009) utilizam o algoritmo Ant Colony combinado com métodos de otimização não linear para desenvolver um novo algoritmo com objetivo de resolver problemas de agendamento contínuo de fabricação de aço.

\section{METODOLOGIA}

Independente do assunto, a ciência no nosso tempo produz em larga escala, então, configura-se uma dificuldade tremenda reunir, sem uma ferramenta adequada para tal, a produção científica de qualquer área ou a que se tem interesse, definindo então o tema e o problema, contidos na introdução desse estudo.

Para dar sustentação ao estudo, é realizada uma revisão bibliográfica acerca do tema. Depois foi realizado um levantamento junto a base Web of Sciences em junho de 2017, com a seguinte pesquisa: "PRODUCTION SCHEDUL*" AND "HEURISTIC*". A busca retornou 699 documentos, dos quais foram processados pelo sistema NAILS (KNUTAS et al., 2015). Os dados gerados pelo sistema são analisados a seguir. A metodologia do trabalho foi baseada na 
proposta por Fahimniaet al. (2015) e Knutaset al. (2015), como demonstrado na Figura 2.

Figura 2: Passos da metodologia

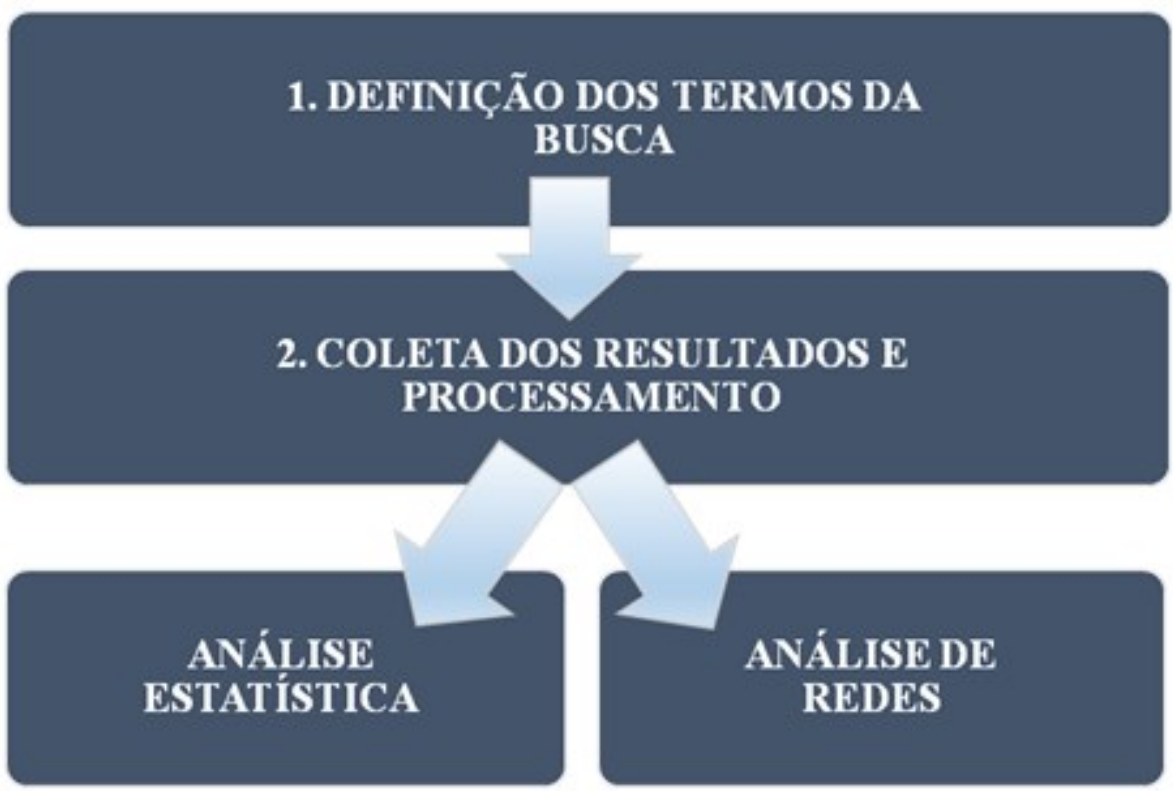

Fonte: Autoria Própria (2017).

\section{RESULTADOS}

Nessa seção os resultados serão apresentados em duas etapas: Primeiro uma análise estatística e depois uma análise de redes.

\section{ANÁLISE ESTATIÍSTICA}

\section{Publicações Por Ano}

A Figura 3 demonstra que apesar de ser uma linha de pesquisa antiga, ainda continua em constante desenvolvimento e interesse, apresentando um crescimento contínuo no número de publicações por ano:

As publicações mais recentes têm focado no desenvolvimento de soluções híbridas, ou seja, na utilização de uma ou mais meta-heurísticas, ou ainda associadas a outras técnicas, como fuzzy, com o objetivo de obter uma melhor solução, como por exemplo: "The Dual-Ants: a novel hybrid approach for the flexible job shop schedulling problem with preventive maintenance", "A hybrid heuristical gorithm for the no-ait flow shops chedulling problem", "A Hybrid Particle swarm Optimization method for permutation flow shop Schedulling" $e$ "Production Schedulling using adaptable fuzzy logic with geneticalgorithms" 
Figura 3: Publicações por ano

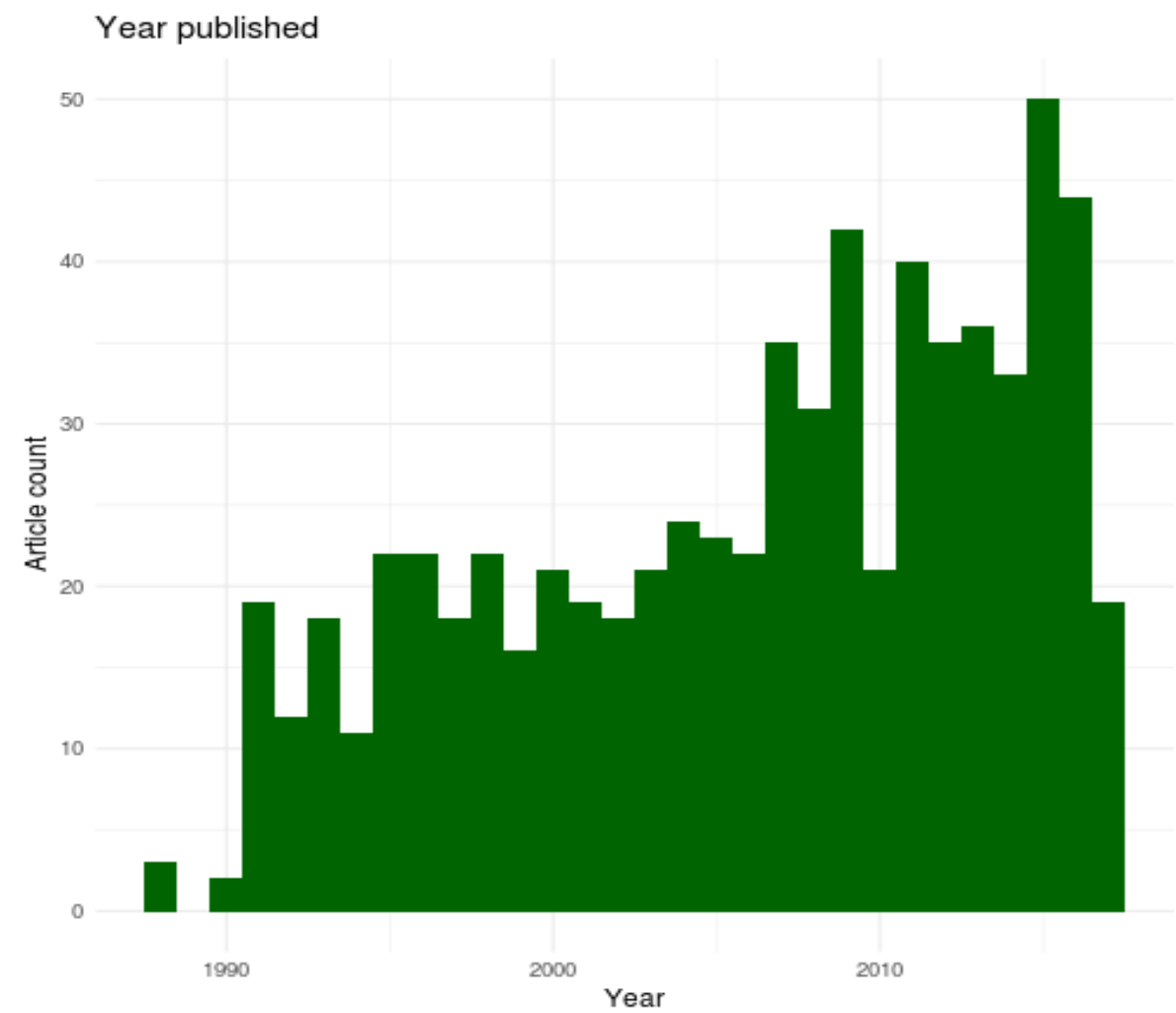

Fonte: Autoria Própria (2017).

\section{Autores Mais Importantes}

A Figura 4 demonstra os autores mais produtivos, ou seja, estão ordenados pelo número de artigos publicados, havendo um maior destaque para LI, WEI com 6 publicações, seguido por DANIELS, RL e CHAN, WT com 5 publicações cada, sendo esses os 3 autores que mais publicaram sobre o tema.

Em uma análise dos autores mais importantes baseado no total de citações recebidas, a figura abaixo Error! Reference source not found. demonstra os autores mais citados, onde o autor DANIELS, RL apresentou um maior destaque, sendo citado mais de 250 vezes, em segundo lugar temos o autor CHANG, YL sendo citado pouco mais de 200 vezes e o terceiro autor mais citado é o HO, JC contabilizando um pouco mais de 150 citações.

Esses gráficos indicam os autores mais populares, como é possível observar, nem sempre o autor mais produtivo é o mais citado e também, como explicado anteriormente, um maior número de citação não necessariamente indica que um autor é mais relevante. 
Figura 4: Autores mais produtivos

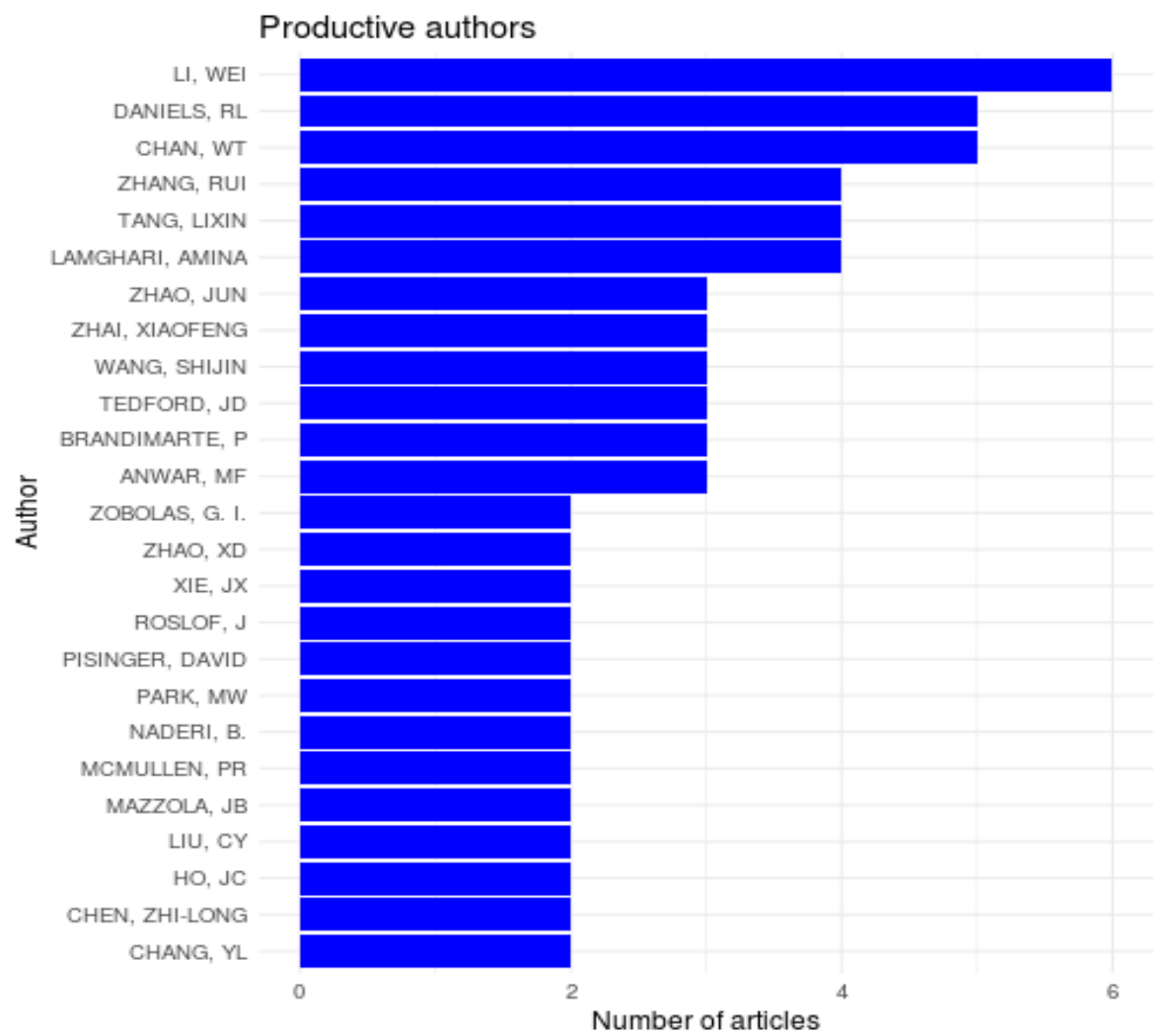

Fonte: Autoria Própria (2017). 
Figura 5: Autores mais citados

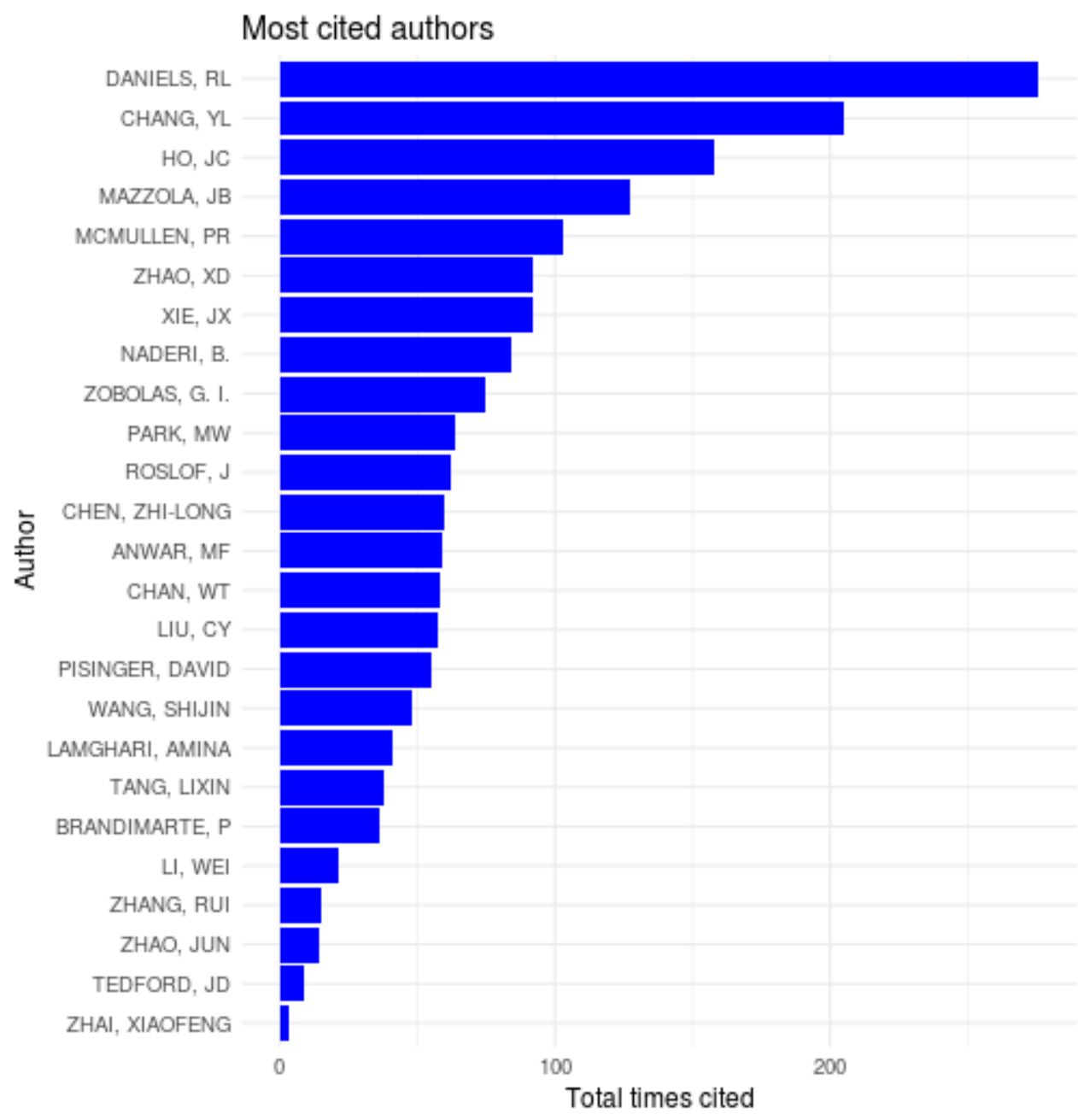

Fonte: Autoria própria (2017).

\section{Periódicos Mais Importantes}

A Figura 6 demonstra os periódicos que mais contribuíram com o tema, ou seja, os que possuam a maior quantidade de artigos publicados (retornados na busca). Os três com um maior destaque são: INTERNATIONAL JOURNAL OF PRODUCTION RESEARCH com pouco mais de 60 publicações, depois temos o EUROPEAN JOURNAL OF OPERATIONAL RESEARCH com 50 publicações e o COMPUTERS \& OPERATIONS RESEARCH com aproximadamente 20 publicações. 
Figura 6: Periódicos mais populares

\section{Most popular publications}

INTERINATIONAL JOURNAL OF PRODUCTION RESEARCH EUROPEAN JOURNAL OF OPERATIONAL RESEARCH COMPUTERS \& OPERATIONS RESEARCH INTERNATIONAL JOURNAL OF ADVANCED MANUFACTURING TE COMPUTERS \& INDUSTRIAL ENGINEERING OPERATIONS RESEARCH INTERNATIONAL JOURNAL OF PRODUCTION ECONOMICS INFORMS JOURNAL ON COMPUTING JOURNAL OF THE OPERATIONAL RESEARCH SOCIETY PRODUCTION PLANNING \& CONTROL IIE TRANSACTIONS

INTERFACES EXPERT SYSTEMS WITH APPLICATIONS COMPUTERS \& CHEMICAL ENGINEERING OMEGA-INTERINATIONAL JOURNAL OF MANAGEMENT SCIENCE MANAGEMENT SCIENCE ANNALS OF OPERATIONS RESEARCH INTERNATIONAL JOURNAL OF INDUSTRIAL ENGINEERING-TH APPLIED SOFT COMPUTING NAVAL RESEARCH LOGISTICS JOURNAL OF INTELLIGENT MANUFACTURING INTERNATIONAL JOURNAL OF OPERATIONS \& PRODUCTION M ENGINEERING OPTIMIZATION INTERNATIONAL JOURNAL OF COMPUTER INTEGRATED MANUF INDUSTRIAL \& ENGINEERING CHEMISTRY RESEARCH

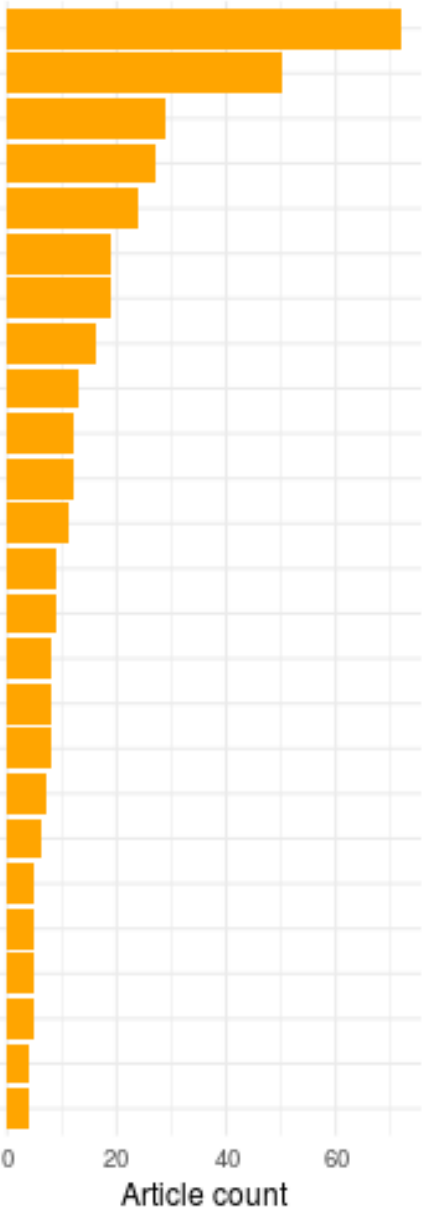

Fonte: Autoria própria (2017).

A Figura 7Error! Reference source not found. demonstra os periódicos mais citados, onde o INTERNATIONAL JOURNAL OF PRODUCTION RESEARCH obteve um maior destaque sendo citado quase 1.000 vezes, depois o EUROPEAN JOURNAL OF OPERATIONAL RESERCH com pouco mais de 900 citações e em seguida o OPERATIONS RESEARCH com quase 750 citações. 
Figura 7: Periódicos mais citados

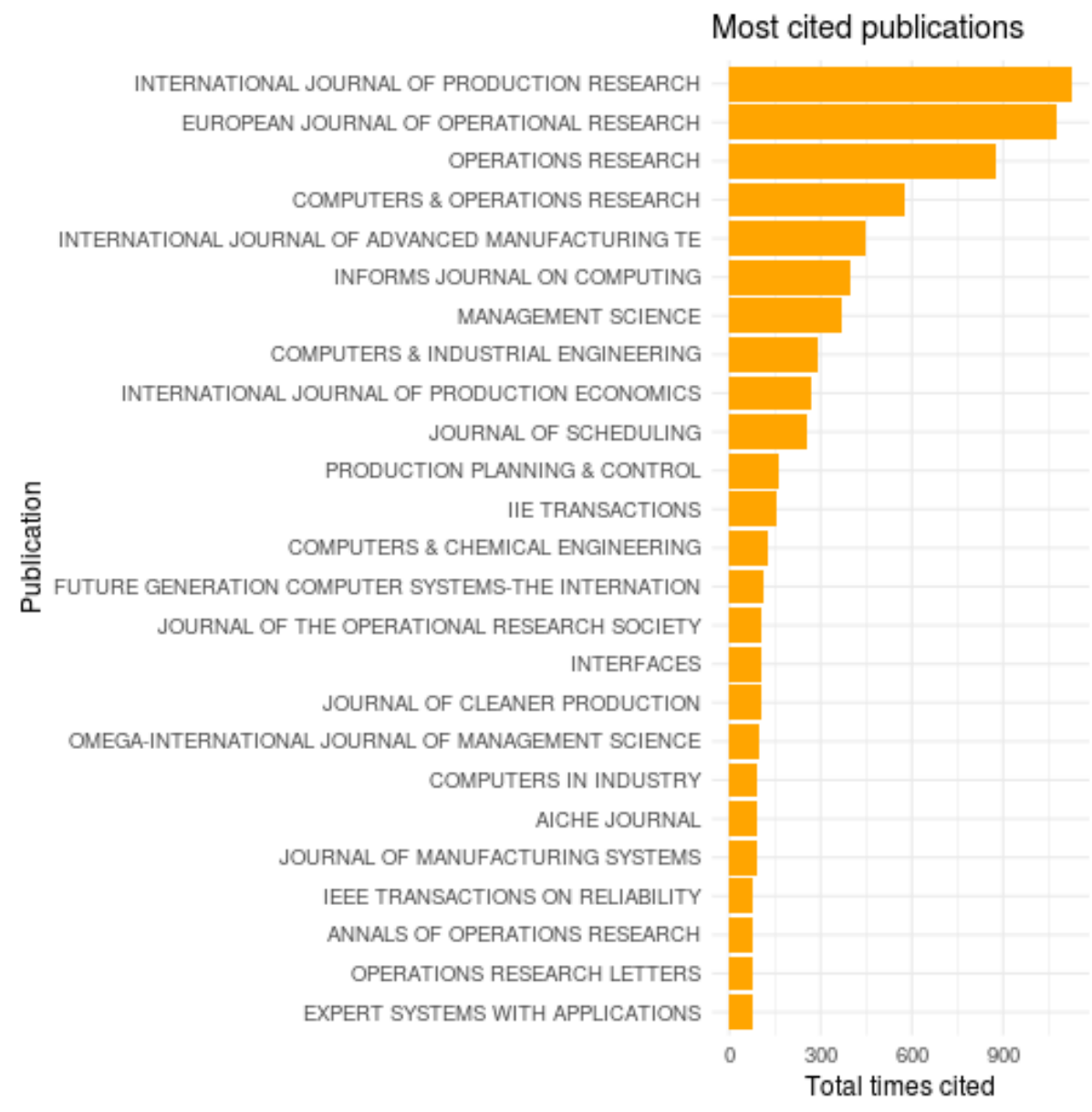

Fonte: Autoria própria (2017).

É importante salientar que os periódicos que obtiveram maior destaque nas Figuras 6 e 7, foram INTERNATIONAL JOURNAL OF PRODUCTION RESEARCH e EUROPEANL JOURNAL OF OPERATIONAL RESEARCH, sendo os com a maior quantidade de publicações e também os mais citados.

\section{ANÁLISE DE REDES}

Os trabalhos mais importantes são identificados no Quadro 1 por três métricas:

$1^{\circ}$ - In-degree na rede de citação;

$2^{\circ}$ - Page Rank;

$3^{\circ}$ - Número de citações, fornecida pela Web of Science; 
Quadro 1: Trabalhos mais importantes, resultantes da busca

\begin{tabular}{|c|c|c|c|}
\hline ARTIGO & InDegree & № Citações & PageRank \\
\hline $\begin{array}{l}\text { | ZIPKIN P, 1991, OPER RES, V39, P56, } \\
\text { DOI 10.1287/OPRE.39.1.56 | }\end{array}$ & 12 & 91 & 0.0001306 \\
\hline $\begin{array}{l}\text { | LOPEZ L, 1998, EUR J OPER RES, V106, } \\
\text { P317, } \\
\text { DOI 10.1016/S0377-2217(97)00277-4 | }\end{array}$ & 10 & 81 & 0.0001363 \\
\hline $\begin{array}{c}\text { | MACCARTHY B, 1993, INT J PROD RES, } \\
\text { V31, P59, } \\
\text { DOI 10.1080/00207549308956713 | }\end{array}$ & 9 & 141 & 0.0001185 \\
\hline $\begin{array}{l}\text { | TANG L, 2002, INT J PROD RES, V40, P55, } \\
\text { DOI 10.1080/00207540110073000 | }\end{array}$ & 8 & 106 & 0.0001094 \\
\hline $\begin{array}{l}\text { | DANIELS R, 1996, MANAGE SCI, V42, } \\
\text { P1260, } \\
\text { DOI 10.1287/MNSC.42.9.1260 | }\end{array}$ & 8 & 44 & 0.0001112 \\
\hline
\end{tabular}

Fonte: Autoria própria (2017).

Os trabalhos identificados na Quadro 2, não estavam no conjunto resultante da busca, onde pode-se observar.

Quadro 2: Trabalhos mais importantes, não presentes no resultado da busca

\begin{tabular}{|c|c|c|}
\hline ARTIGO & InDegree & PageRank \\
\hline MAWAZ M, 1983, OMEGA-INT J & & 0.0002019 \\
\hline 0483(83)90088-9 & 46 & 0.0002462 \\
\hline $\begin{array}{c}\text { BAKER K R, 1974, INTRO } \\
\text { SEQUENCING SCH }\end{array}$ & 45 & 0.0002140 \\
\hline GAREY M.R., 1979, COMPUTERS \\
INTRACTABI
\end{tabular}

Fonte: Autoria própria (2017).

A seguir é mostrada a rede de co-citações, ou seja, essa rede contém todas as referências dos artigos selecionados, sendo gerada com auxílio do Gephi: 
Figura 8: Rede de Co-citações, com a utilização do PageRank

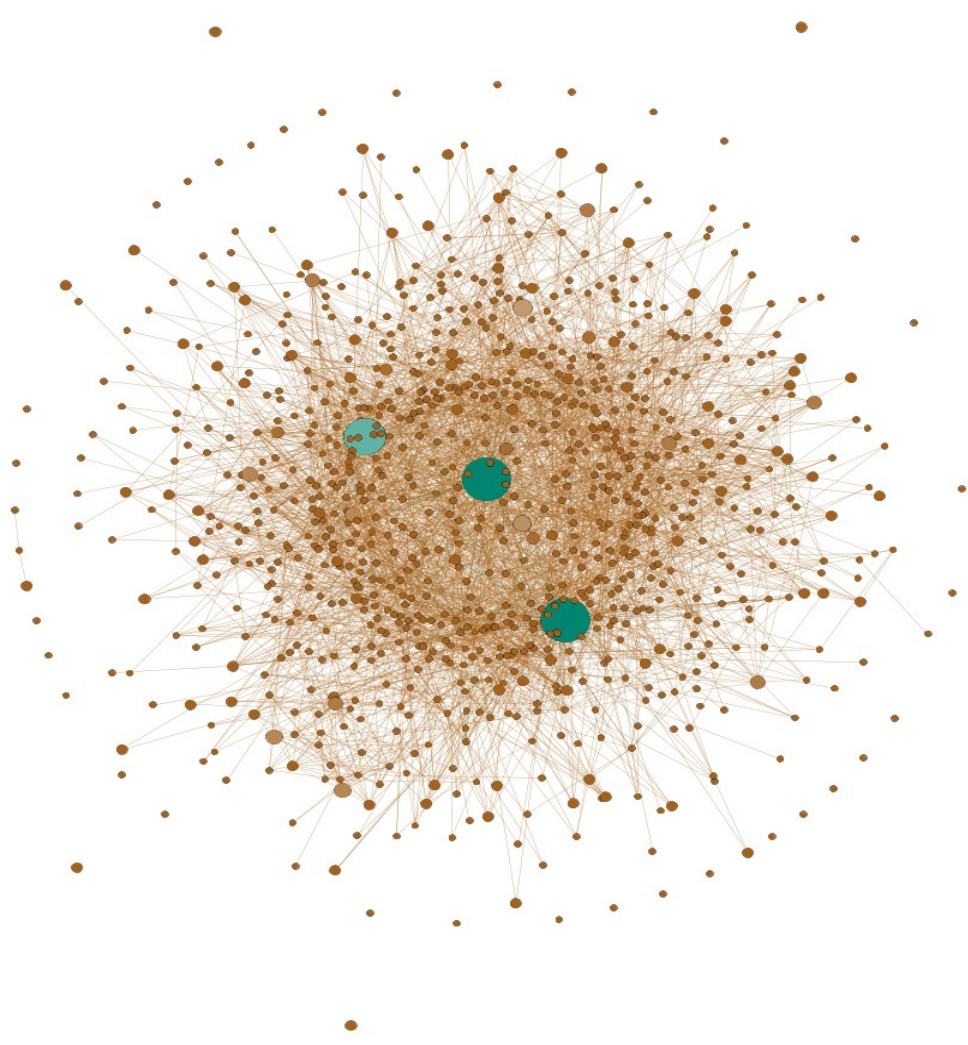

Fonte: Autoria Própria (2017).

Após a importação dos dados da rede pelo Gephi, foi aplicada a métrica Page Rank, onde o maior grau é associado a uma cor verde, além da circunferência dos nós. Como essa rede possuía um grande volume de dados, e para que fosse possivel realizar uma análise visual, sua representação gráfica foi feita com apenas $8 \%$ dos nós (artigos) e 18\% dos arcos (citações) da rede original, dessa forma, podemos destacar os trabalhos:

- NAWAZ M, 1983, OMEGA-INT J MANAGE S, V11, P91, DOI 10.1016/0305-0483(83)90088-9;

- GAREY M.R., 1979, COMPUTERS INTRACTABI;

- $\quad$ BAKER K R, 1974, INTRO SEQUENCING SCH.

$O$ artigo que mais se destacou, obtendo um maior PageRank foi " $A$ heuristic algorithm for the m-machine, n-jobflow-shop sequencing problem" de Nawaz, Enscore e Ham(1983), no qual é proposto um algoritmo para o problema de sequenciamento do tipo Flow-Shop para $\mathrm{m}$ máquinas e $n$ Jobs, onde os resulados obtidos foram comparados com outros 15 algoritmos.

Um trabalho mais antigo que também houve destaque é o "Optimal twoand three-stage production schedules with setup times included" de Johnson (1954), em que é descrita uma regra de sequenciamento muito difundida (Regra de Johnson), onde cada máquina pode lidar com apenas um item por vez e cada item deve ser processado através da máquina um e depois da máquina dois(JOHNSON, 1954). 
Goldberg (1989) em um trabalho mais recente trata a meta-heurística de Algoritmos Genéticos, estando em destaque entre os trabalhos com um maior PageRank.

\section{CONCLUSÃO}

Este trabalho demonstrou uma revisão de mapeamento sistemático com relação à Programação da Produção e suas principais meta-heurísticas. Apesar de ser uma área antiga, com seu surgimento por meados da década de 50, ainda apresenta um crescimento. Devido ao grande número de trabalhos, torna-se complexa a análise dos dados, o que foi possível somente com a utilização da ferramenta NAILS para o processamento e geração de gráficos, dados em formas tabulares e grafos através do GEPHI.

O número de citações tem sido utilizado por muitos anos para análise, por exemplo, de autores e trabalhos mais relevantes para um determinado campo de pesquisa, todavia, essa métrica apresenta algumas deficiências e parte delas são supridas pelo PageRank.

Dessa forma, buscou-se através dessa métrica elencar os trabalhos mais relevantes com relação às meta-heurísticas utilizadas na programação da produção, possibilitando ao desenvolvimento de revisões bibliográficas futuras e suporte a outros pesquisadores.

Através dessa pesquisa foi possível observar, que esse campo de pesquisa se mantém em constante desenvolvimento, e que trabalhos mais recentes buscam a utilização de soluções híbridas com a utilização meta-heurísticas em conjunto ou com outras técnicas como redes neurais e Fuzzy.

Conclui-se que as ferramentas utilizadas contribuíram para o desenvolvimento desse trabalho, pois reduziu de forma significante o trabalho de coleta e análise de dados, o que antes se tratava de uma tarefa árdua e sujeita erros. Diante das análises feitas e para a elaboração de trabalhos futuros deve-se fazer um acompanhamento constante desse campo e com isso um estudo mais detalhado dos trabalhos mais recentes. 


\title{
PRODUCTION AND META-HEURISTIC PROGRAMMING: A REVIEW OF SYSTEMATIC MAPPING
}

\begin{abstract}
Production Programming has received attention from researchers in the area of Operational Research since the 1950s and a large number of papers have been developed since then, similarly due to the Internet, the bibliographic tracking of a field of research is taking longer, as well as to find relationships between authors and relevant publications, is becoming increasingly difficult due to the growing volume of information. (Climbing, Simulated Annealing, Tabu Search, Harmony Search, GRASP, Evolutionary Algorithms, Genetic Algorithms, Evolution Strategies, Genetic Programming, Differential Evolution, Evolutionary Programming, Particle Swarm Optimization, Memetic Algorithms and Ant Colony), through bibliometric indicators and analysis of networks (Page Rank), it is tried to demonstrate the relationship of articles and authors and more prestigious publications of data originated from the Web of Science base with the aid of the tools NAILS and GEPHI. Finally, it is demonstrated that the use of metaheuristics in Production Programming, despite being an old line of research, still remains with great interest and in constant development.
\end{abstract}

KEYWORDS: Production Scheduling. Meta-Heuristics. Network Analysis. 


\section{REFERÊNCIAS}

ARROYO, J. E. C. Heurísticas E Metaheurísticas Para Multiobjetivo. Campinas, SP, Brasil: Universidade Estadual de Campinas, 2002.

ATIGHEHCHIAN, A.; BIJARI, M.; TARKESH, H. A novel hybrid algorithm for scheduling steel-making continuous casting production. Computers and Operations Research, v. 36, n. 8, p. 2450-2461, 2009. crossref

BAGLEY, J. D. The behavior of adaptive systems which employ genetic and correlation algorithms. Bethesda, Maryland, EUA: The University of Michigan, 1967.

BARRICELLI, N. A. Numerical testing of evolution theories - Part I Theoretical introduction and basic tests. Acta Biotheoretica, v. 16, n. 1-2, p. 69-98, 1962. crossref

BRIN, S.; PAGE, L. The anatomy of a large scale hypertextual Web search engine. Computer Networks and ISDN Systems, v. 30, n. 1/7, p. 107-17, 1998.

DING, Y. Topic-based PageRank on author cocitation networks. Journal of the American Society for Information Science and Technology, v. 14, n. 4, p. n/a-n/a, 2011a.

DING, Y. Applying weighted PageRank to author citation networks. Journal of the American Society for Information Science and Technology, v. 62, n. 2, p. 236245, fev. 2011b. crossref

DING, Y.; CRONIN, B. Popular and/or prestigious? Measures of scholarly esteem. Information Processing and Management, v. 47, n. 1, p. 80-96, 2011. crossref

DORIGO; M. Optimization, Learning and Natural Algorithms. Ph.D. Thesis, Politecnico di Milano, Italy, 1992.

EBERHART, R.; KENNEDY, J. A new optimizer using particle swarm theory. MHS'95. Proceedings of the Sixth International Symposium on Micro Machine and Human Science. Anais...IEEE, 1995Disponível em: <http://linkinghub.elsevier.com/retrieve/pii/S0038092X16305321\%0Ahttp://ieee xplore.ieee.org/document/4475427/\%0Ahttp://ieeexplore.ieee.org/lpdocs/epic0 3/wrapper.htm?arnumber=6714417\%0Ahttp://dx.doi.org/10.1016/j.energy.2014 .07.001\%0Ahttp://dx.doi.org/10.10>crossref 
EHRGOTT, M.; GANDIBLEUX, X. A survey and annotated bibliography of multiobjective combinatorial optimization. OR Spectrum, v. 22, n. 4, p. 425-460, 2000. crossref

FAHIMNIA, B.; SARKIS, J.; DAVARZANI, H. Green supply chain management: A review and bibliometric analysis. [s.I.] Elsevier, 2015. v. 162

FEO, T. A.; BARD, J. F. Flight Scheduling and Maintenance Base Planning. Management Science, v. 35, n. 12, p. 1415-1432, 1989. crossref

FEO, T. A.; RESENDE, M. G. C. Greedy Randomized Adaptive Search Procedures. Journal of Global Optimization, v. 6, n. 2, p. 109-133, 1995. crossref

FEO, T. A.; VENKATRAMAN, K.; BARD, J. F. A GRASPTM for a difficult single machine scheduling problem. Computers \& Operations Research, v. 18, n. 8, p. $635-643,1991$. crossref

FRASER, A. S. Simulation of genetic systems by automatic digital computers. Australian Journal of Biological Sciences, v. 10, n. 4, p. 484-491, 1957. crossref

GARFIELD, E. Citation Indexes for Science: A New Dimension in Documentation through Association of Ideas. Science, v. 122, n. 3159, p. 108 LP-111, 15 jul. 1955.

GEISMAR, J. H. N. et al. The integrated production and transportation scheduling problem for a product with a short lifespan. INFORMS Journal on Computing, $v$. 20, n. 1, p. 21-33, 2008. crossref

GLOVER, F. Future paths for integer programming and links to artificial intelligence. Computers and Operations Research, v. 13, n. 5, p. 533-549, 1986. crossref

GLOVER, F.; LAGUNA, M.; MARTI, R. Principles of tabu search. Approximation Algorithms and Metaheuristics, v. 23, p. 1-12, 2007.

GLOVER, F.; MARTí, R. Tabu Search. Tabu Search, p. 1-16, 1986.

GOLDBERG, D. E. Genetic Algorithms in Search, Optimization and Machine Learning. 1st. ed. Boston, MA, USA: Addison-Wesley Longman Publishing Co., Inc., 1989. 
HEATH, S. K.; BARD, J. F.; MORRICE, D. J. A GRASP for simultaneously assigning and sequencing product families on flexible assembly lines. Annals of Operations Research, p. 1-29, 2012.

HICKS, C.; SONG, D. P.; EARL, C. F. Dynamic scheduling for complex engineer-toorder products. International Journal of Production Research, v. 45, n. 15, p. 3477-3503, 2007. crossref

JOHNSON, S. M. Optimal two- and three-stage production schedules with setup times included. Naval Research Logistics Quarterly, v. 1, n. 1, p. 61-68, 1954. crossref

KENNEDY, J.; EBERHART, R. Particle swarm optimization. 1995 IEEE International Conference on Neural Networks (ICNN 95), v. 4, p. 1942-1948, 1995. crossref

KHABSA, M.; GILES, C. L. The number of scholarly documents on the public web. PLoS ONE, v. 9, n. 5, 2014. crossref

KHAN, A.; NIEMANN-DELIUS, C. Production scheduling of open pit mines using particle swarm optimization algorithm. Advances in Operations Research, v. 2014, 2014.

KLINK, S. et al. Within Bibliographical Data. p. 234-243, 2006.

KNUTAS, A. et al. Cloud-based Bibliometric Analysis Service for Systematic Mapping Studies. Proceedings of the 16th International Conference on Computer Systems and Technologies, p. 184-191, 2015. crossref

LIU, B.; WANG, L.; JIN, Y.-H. An effective hybrid particle swarm optimization for no-wait flow shop scheduling. The International Journal of Advanced Manufacturing Technology, v. 31, n. 9-10, p. 1001-1011, 2007. crossref

LIU, R. et al. A multipopulation PSO based memetic algorithm for permutation flow shop scheduling. The Scientific World Journal, v. 2013, 2013.

MACCARTHY, B. L.; LIU, J. Addressing the gap in scheduling research: a review of optimization and heuristic methods in production scheduling. International Journal of Production Research, v. 31, n. 1, p. 59-79, 1993. crossref

MASLOV, S.; REDNER, S. Promise and Pitfalls of Extending Google's PageRank Algorithm to Citation Networks. Journal of Neuroscience, v. 28, n. 44, p. 1110311105,2008 . crossref 
MENA-CHALCO, J. P.; JUNIOR, R. M. C. scriptLattes: an open-source knowledge extraction system from the Lattes platform. Journal of the Brazilian Computer Society, v. 15, n. 4, p. 31-39, 2009. crossref

MILLER, D. M. et al. A hybrid genetic algorithm for the single machine scheduling problem. Journal of Heuristics, v. 5, n. 4, p. 437-454, 1999. crossref

MONKMAN, S. K.; MORRICE, D. J.; BARD, J. F. A production scheduling heuristic for an electronics manufacturer with sequence-dependent setup costs. European Journal of Operational Research, v. 187, n. 3, p. 1100-1114, 2008. crossref

MOSCATO, P.; COTTA, C. Una introduccion a los algoritmos memeticos. Revista Iberoamericana de Inteligencia Artificial, v. 19, n. June 2017, p. 131-148, 2003.

NAWAZ, M.; ENSCORE, E. E.; HAM, I. A heuristic algorithm for the m-machine, njob flow-shop sequencing problem. Omega, v. 11, n. 1, p. 91-95, 1983. crossref

OTTE, E.; ROUSSEAU, R. Social network analysis: a powerful strategy, also for the information sciences. Journal of Information Science, v. 28, n. 6, p. 441-453, 2002. crossref

PETERSEN, K.; VAKKALANKA, S.; KUZNIARZ, L. Guidelines for conducting systematic mapping studies in software engineering: An update. Information and Software Technology, v. 64, p. 1-18, 2015. crossref

PICKARDT, C. et al. Generating dispatching rules for semiconductor manufacturing to minimize weighted tardiness. Proceedings - Winter Simulation Conference, p. 2504-2515, 2010. crossref

RAAYMAKERS, W. H. M.; HOOGEVEEN, J. A. Scheduling multipurpose batch process industries with no-wait restrictions by simulated annealing. European Journal of Operational Research, v. 126, n. 1, p. 131-151, 2000. crossref

RUSSELL, S. J.; NORVIG, P. Artificial Intelligence: A Modern Approach. 3. ed. Englewood Cliffs, New Jersey - EUA: Prentice Hall, 1995.

SCHWEFEL, H.-P. Evolution and Optimum Seeking. Sixth-generation computer technology series, n. October, p. ix, 444, 1995. 
SCHWEFEL, H.-P.; BÄCK, T. Artificial evolution: how and why? In: QUAGLIARELLA, D. et al. (Eds.). . Genetic Algorithms and Evolution Strategy in Engineering and Computer Science: Recent Advances and Industrial Applications. 1. ed.

Chichester: Wiley, 1998.

SHAO, W.; PI, D. A self-guided differential evolution with neighborhood search for permutation flow shop scheduling. Expert Systems with Applications, v. 51, p. 161-176, 2016. crossref

STORN, R.; PRICE, K. Differential Evolution - A Simple and Efficient Heuristic for global Optimization over Continuous Spaces. Journal of Global Optimization, v. 11, n. 4, p. 341-359, 1997. crossref

TANG, L.; WANG, X.; LIU, J. Color-coating production scheduling for coils in inventory in steel industry. IEEE Transactions on Automation Science and Engineering, v. 5, n. 3, p. 544-549, 2008. crossref

VIDAL, J. C. et al. Machine scheduling in custom furniture industry through neuroevolutionary hybridization. Applied Soft Computing Journal, v. 11, n. 2, p. 16001613, 2011. crossref

WAGNER, H. M.; WHITIN, T. M. Dynamic Version of the Economic Lot Size Model. Management Science, v. 50, n. 12_supplement, p. 1775-1777, dez. 1958.

WANG, L.; PAN, Q. K.; TASGETIREN, M. F. Minimizing the total flow time in a flow shop with blocking by using hybridm harmony search algorithms. Expert Systems with Applications, v. 37, n. 12, p. 7929-7936, 2010. crossref

WASSERMAN, S.; FAUST, K. Social network analysis : methods and applications. [s.l.] Cambridge University Press, 1994. crossref

WEISE, T. Global Optimization Algorithms - Theory and Application. p. 1223, 2011.

YALAOUI, N. N. et al. Fuzzy Project Scheduling. In: KAHRAMAN, C.; YAVUZ, M. (Eds.). . Production Engineering and Management under Fuzziness. Berlin, Heidelberg: Springer Berlin Heidelberg, 2010. v. 252p. 143-170. crossref

YAN, E.; DING, Y. Weighted citation: An indicator of an article's prestige. Journal of the American Society for Information Science and Technology, 2010. crossref 
ZONG WOO GEEM; JOONG HOON KIM; LOGANATHAN, G. V. A New Heuristic Optimization Algorithm: Harmony Search. Simulation, v. 76, n. 2, p. 60-68, 2001. crossref

Av. Aluizio da Silva Gomes, 50, Bairro Granja dos Cavaleiros, Macaé, Rio de Janeiro, Brasil.

Direito autoral: Este artigo está licenciado sob os termos da Licença Creative Commons-Atribuição 4.0 Internacional.

\section{(c) (1)}

